\title{
Reversible Valproate Induced Pisa Syndrome and Parkinsonism in a Neuro-Oncology Patient with Depression and Epilepsy
}

\author{
Andrea Botturi ${ }^{\mathrm{a}, \mathrm{b}} \quad$ Antonio Silvani $^{\mathrm{a}} \quad$ Gabriella Pravettoni ${ }^{\mathrm{d}} \mathrm{e}$ \\ Riccardo Augusto Paoli ${ }^{\mathrm{b}}$ Claudio Lucchiaric \\ ${ }^{a}$ Department of Neuro-Oncology, Fondazione IRCCS Istituto Neurologico Carlo Besta, \\ Milan, Italy; ${ }^{b}$ Department of Psychiatry, Università degli Studi di Milano, Fondazione \\ IRCCS Ca'Granda Ospedale Maggiore Policlinico, Milan, Italy; ' Department of Philosophy, \\ Università degli Studi di Milano, Milan, Italy; ${ }^{\mathrm{d}}$ Department of Oncology and \\ Hemato-Oncology, Università degli Studi di Milano, Milan, Italy; ${ }^{\mathrm{e}}$ Applied Research Unit \\ for Cognitive and Psychological Science, European Institute of Oncology, Milan, Italy
}

\section{Keywords}

Parkinsonism · Valproic acid · Depression · Epilepsy · Neuro-oncology

\begin{abstract}
Neurological and psychiatric conditions frequently overlap in neuro-oncology. This overlapping negatively affects patients' quality of life and decreases the ability of providers to manage specific symptoms by therapy modulation, especially when psychopharmacotherapy needs to be prescribed. We describe here a patient with recurrent brain tumor, symptomatic epilepsy and depression who developed Pisa syndrome and parkinsonism after several months of valproic acid use. An accurate recognition of symptoms and treatment side effect allowed an appropriate clinical approach so as to rapidly improve both movement disorder and depression without increasing the risk of developing seizure. This has improved the autonomy and quality of life in a patient with poor prognosis.




\section{Case Reports in Neurology}

Botturi et al.: Reversible Valproate Induced Pisa Syndrome and Parkinsonism in a NeuroOncology Patient with Depression and Epilepsy

\section{Case Report}

A 59-year-old inpatient was admitted to the neurological cancer center Istituto Neurologico 'Carlo Besta' for worsened condition. He had been diagnosed 3 years before with recurrent high-grade glioma and underwent two surgeries, radiotherapy plus chemotherapy. He suffered from epilepsy in therapy with levetiracetam. Since this treatment was poorly tolerated for dysphoric status, it was changed to valproic acid (VPA) (900 mg/day). However, 6 months before hospitalization - 3 after the new antiepileptic drug -, during periodical outpatient clinical evaluation, a depressed mood emerged, with work retirement, somatization, loss of weight, and sleep disorder. Furthermore, cognitive impairment seemed to be present, with short-term memory deficit and dyscalculia. The psychopathological history of the patient was characterized by the presence of hyperthymic temperament and subsyndromal hypomania. A previous depressive episode had been reported during young adulthood.

Six months before hospitalization, he started taking paroxetine $(20 \mathrm{mg} /$ day $)$, with slight improvement of mood at clinical global impression. The patient needed to be hospitalized, due to gait and upright posture disturbances. Brain magnetic resonance imaging (fig. 1) showed removal of bulky lesion located in the right parietal peritrigonal region. There was marked dilatation of the temporal horn, the third ventricle and the left lateral ventricle. Widespread signal alteration was prevalent in the white matter and in the splenium. After administration of gadolinium, it was not possible to observe clear signs of dissemination in the supratentorial and infratentorial region.

The electroencephalogram described rather unstable background activity, better on the left hemisphere, slower and more unstable on the right. Multifocal epileptiform abnormalities were observed in the right hemisphere. On the clinical examination, the patient showed abnormal posture with sustained lateral bending of the trunk along the sagittal plane on the right side. At the same time, bradykinesia and rigidity of the four limbs were present. Postural instability and falling were present as well. Tendon reflexes were brisk without pyramidal signs. The patient also showed tremor of the voice and hypophonia, hypomimia, short shuffling steps, trouble starting to walk, and difficulty in turning. The signs were symmetrical.

The blood level of VPA was $63 \mu \mathrm{g} / \mathrm{ml}$ (normal value $=40-100$ ), and ammonemia was 57 $\mu \mathrm{g} / \mathrm{dl}$ (normal value $=27-102$ ). During the hospitalization, a pharmacological change was decided that involved tapering off VPA and getting on therapy with lamotrigine (LMT) up to a dose of $200 \mathrm{mg} /$ day, with blood level $7.2 \mathrm{mg} / \mathrm{l}$ (normal value $=3-14$ ). The patient was discharged with a partial regression of symptoms. In the following weeks, neurological and psychiatric condition improved. (table 1)

\section{Discussion}

Depression accounts for $15-20 \%$ of patients with glioma within 8 months after diagnosis [1], even if various longitudinal studies have shown significant improvements in the following months. The precise cause of the depression remains unclear. Previous medical history of depression may predict an increased risk of depression in glioma. Untreated depression is an established risk factor for mortality in cancer and neurological disease [2].

Selective serotonin reuptake inhibitors (SSRIs) are associated with improvements in disability and neurological impairment after cerebrovascular disease. Conflicting data are 
available with regard to glioma patients. Extrapyramidal symptoms (EPS) have been reported with different classes of antidepressants; they seem not to be dose-related and to be due to short-term or long-term use. The literature concerning SSRIs is controversial, because not the class in itself is responsible for EPS but the patient's own sensitivity. In any case, an increase of 5HT, due to the SSRIs reuptake inhibition, can reduce the dopaminergic tone at basal ganglia level (inducing EPS), as well as at the anterior cingulate and frontal cortex, potentially inducing emotional blunting [3]. However, SSRIs are currently used in Parkinson disease and may improve depression [4].

Epilepsy occurs in about 40-60\% of patients with high-grade glioma, and is itself an unfavorable prognostic factor [5]. Alper et al. [6] performed a large meta-analysis on seizure risk and found an increased risk linked to the use of the antidepressants bupropion and clomipramine. Most antidepressants included in this study showed no association with an increased seizure risk in therapeutic doses, and some were associated with a reduced risk [2]. Furthermore, a systematic revision of the Cochrane database did not appear to show any significant worsening of seizures [7].

VPA is a well-known antiepileptic drug indicated for the prevention of affective episodes [8]. VPA was identified as one of the drugs capable of inducing parkinsonism in elderly patients in the French Pharmacovigilance Database. A similar association was found in a recent literature review [9]. Most cases were shown to be reversible upon VPA discontinuation, although no predictors of reversibility were identified. VPA acts as mild inhibitor of cytochrome enzyme system (CYP450) and can induce pharmacokinetic interactions with drugs metabolized by CYP2D6 and CYP2C9 (e.g. SSRIs) with increase of plasma levels [10].

EPS refers to a condition of sustained lateral bending of the trunk, associated or not with rotation of the spine along the sagittal plane. It was originally described by Ekbom et al. [11] in 1972 in patients on psychiatric drugs.

LMT is often used with severe epilepsy (focal and general onset seizures) [12], and is recommended in bipolar maintenance when depression is prominent. It also plays a role in treating acute bipolar and unipolar depression, for intrinsic antidepressant activity [13]. Some papers have described the use of LMT in hard to treat depression $[14,15]$ due to the weak CYP450-mediated metabolic interaction with SSRIs.

Consistently with the literature, tapering and changing of antiepileptic drugs with LMT has improved neurological and psychiatric symptoms. The choice to remove VPA was due to a less significant EPS (percentage and severity) compared to what is reported in association with antidepressants. The scores of the psychometric scales showed a decrease of depression and disability, with recovery of daily living activity.

\section{Conclusion}

Neurological and psychiatric conditions frequently overlap in neuro-oncology. This overlapping negatively affects patients' quality of life and decreases the ability of providers to manage specific symptoms by therapy modulation, especially when psychopharmacotherapy needs to be prescribed.

In the present case, an oncology patient suffered from depression and epilepsy. However, an accurate recognition of symptoms and treatment side effects allowed an appropriate clinical approach so as to rapidly improve both movement disorder and depression without increasing the risk of developing seizure. This improved the autonomy and quality of life in a patient with poor prognosis. 
Botturi et al.: Reversible Valproate Induced Pisa Syndrome and Parkinsonism in a NeuroOncology Patient with Depression and Epilepsy

\section{Statement of Ethics}

The patient had the opportunity to discuss with his physician in charge the opportunity to publish his clinical case without revealing any personal data in order to share and improve clinical knowledge and practice. The patient signed an inform consent and gave us the authorization to publish his case. Authors guaranteed the anonymity of the patient.

\section{Disclosure Statement}

The authors declare that the study presented received no financial support. The authors declare no conflict of interest.

\section{References}

1 Rooney AG, McNamara S, Mackinnon M, Fraser M, Rampling R, Carson A, Grant R: Frequency, clinical associations, and longitudinal course of major depressive disorder in adults with cerebral glioma. J Clin Oncol 2011;29:4307-4312.

-2 Rooney AG, Brown PD, Reijneveld JC, Grant R: Depression in glioma: a primer for clinicians and researchers. J Neurol Neurosurg Psychiatry 2014;85:230-235.

-3 Opbroek A, Delgado PL, Laukes C, McGahuey C, Katsanis J, Moreno FA, Manber R: Emotional blunting associated with SSRI-induced sexual dysfunction. Do SSRIs inhibit emotional responses? Int J Neuropsychopharmacol 2002;5:147-151.

$\checkmark 4$ Dell'Agnello G, Ceravolo R, Nuti A, Bellini G, Piccinni A, D'Avino C, Dell'Osso L, Bonuccelli U: SSRIs do not worsen Parkinson's disease: evidence from an open-label, prospective study. Clin Neuropharmacol 2001;24:221-227.

5 Vecht CJ, Kerkhof M, Duran-Pena A: Seizure prognosis in brain tumors: new insights and evidencebased management. Oncologist 2014;19:751-759.

6 Alper K, Schwartz KA, Kolts RL, Khan A: Seizure incidence in psychopharmacological clinical trials: an analysis of Food and Drug Administration (FDA) summary basis of approval reports. Biol Psychiatry 2007;62:345-354.

7 Maguire MJ, Weston J, Singh J, Marson AG: Antidepressants for people with epilepsy and depression. Cochrane Database Syst Rev 2014;12:CD010682.

-8 Lambert PA, Carraz G, Borselli S, Carbel S: Neuropsychotropic action of a new anti-epileptic agent: depamide. Ann Med Psychol (Paris) 1966;124:707-710.

-9 Nanau RM, Neuman MG: Adverse drug reactions induced by valproic acid. Clin Biochem 2013;46:13231338.

10 Madhusoodanan S, Velama U, Parmar J, Goia D, Brenner R: A current review of cytochrome P450 interactions of psychotropic drugs. Ann Clin Psychiatry 2014;26:120-138.

11 Ekbom K, Lindholm H, Ljungberg L: New dystonic syndrome associated with butyrophenone therapy. Z Neurol 1972;202:94-103.

12 Matsuo F, Bergen D, Faught E, Messenheimer JA, Dren AT, Rudd GD, Lineberry CG: Placebo-controlled study of the efficacy and safety of lamotrigine in patients with partial seizures. U.S. Lamotrigine Protocol 0.5 Clinical Trial Group. Neurology 1993;43:2284-2291.

13 Reid JG, Gitlin MJ, Altshuler LL: Lamotrigine in psychiatric disorders. J Clin Psychiatry 2013;74:675684.

14 Bruno A, Micò U, Pandolfo G, Mallamace D, Abenavoli E, Di Nardo F, D’Arrigo C, Spina E, Zoccali RA, Muscatello MR: Lamotrigine augmentation of serotonin reuptake inhibitors in treatment-resistant obsessive-compulsive disorder: a double-blind, placebo-controlled study. J Psychopharmacol 2012;26:1456-1462.

15 Chang CM, Sato S, Han C: Evidence for the benefits of nonantipsychotic pharmacological augmentation in the treatment of depression. CNS Drugs 2013;27(suppl 1):S21-S27. 


\section{Case Reports in Neurology}

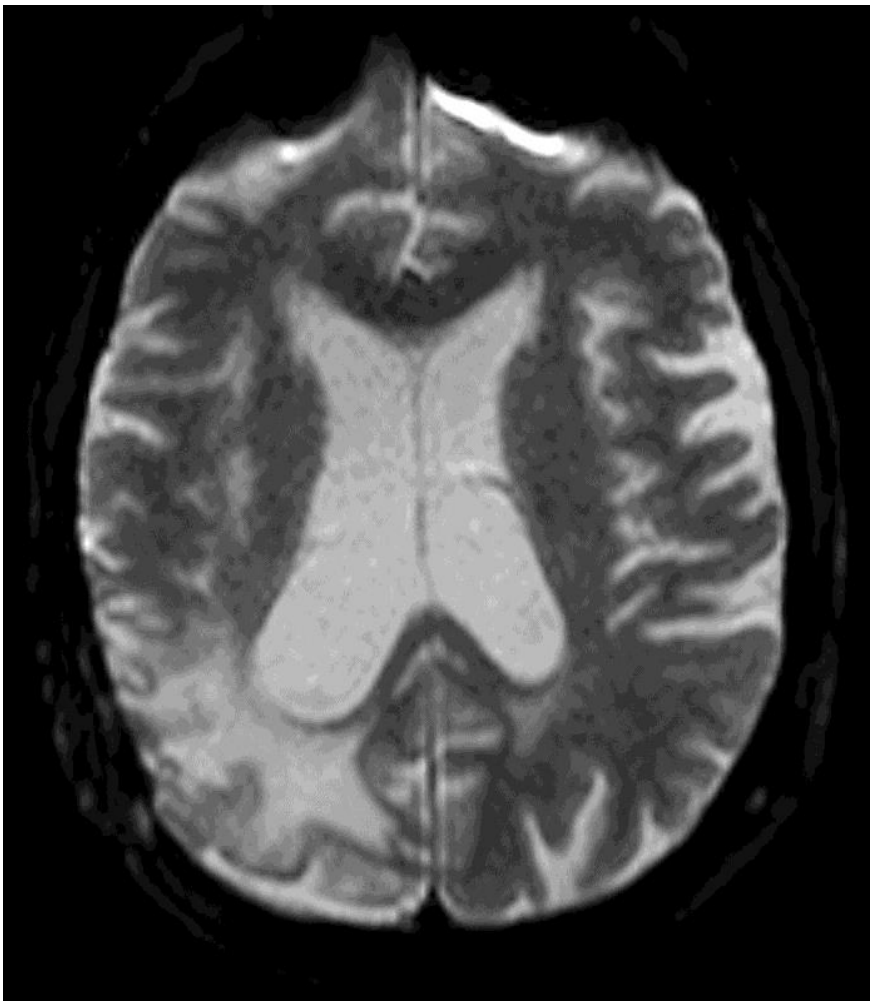

Fig. 1. Magnetic resonance image of the brain.

Table 1. Clinical characteristics over time

\begin{tabular}{lccc}
\hline & T0 & T1 & T2 \\
\hline UPDRS III & $52 / 108$ & $7 / 108$ & $0 / 108$ \\
MMSE & $25 / 30$ & $27 / 30$ & $29 / 30$ \\
Clock Drawing Test & 1 & 1 & 1 \\
HAM-D & 25 & 4 & 3 \\
MADRS & 32 & 10 & 6 \\
ADL & $5 / 6$ & $6 / 6$ & $6 / 6$ \\
IADL & $1 / 8$ & $6 / 8$ & $8 / 8$ \\
Barthel Index & $70 / 100$ & $100 / 100$ & $100 / 100$ \\
\hline
\end{tabular}

$\mathrm{T} 0=$ On admission to hospital; $\mathrm{T} 1=1$ month later; $\mathrm{T} 2=3$ months later; UPDRS = Unified Parkinson Disease Rating Scale (part III motor examination); MMSE = Mini Mental State Evaluation; HAM-D = Hamilton Rating Scale for Depression; MADRS = Montgomery Asberg Depression Rating Scale; ADL = Activities of Daily Living; IADL = Instrumental Activities of Daily Living. 